\title{
Sexismo, pensamientos distorsionados y violencia en las relaciones de pareja en estudiantes universitarios de Ecuador de áreas relacionadas con el bienestar y la salud
}

Sexism, Distorted Thoughts and Violence in Couple Relationships in Ecuadorian Universities with Students Related to Welfare and Health

\author{
SANTIAGO BOIRA ${ }^{\mathrm{a}}$ \\ Universidad de Zaragoza, España \\ ORCID: http://orcid.org/0000-0001-5073-4465 \\ Elisa Chilet-Rosell \\ Universidad de Cuenca, Ecuador \\ Sofía Jaramillo-Quiroz \\ Universidad de Cuenca, Ecuador \\ Jessica Reinoso \\ Universidad de Cuenca, Ecuador
}

a Autor de correspondencia. Correo electrónico: sboira@unizar.es

Para citar este artículo: Boira, S., Chilet-Rosell, E., Jaramillo-Quiroz, S., \& Reinoso, J. (2017). Sexismo, pensamientos distorsionados y violencia en las relaciones de pareja en estudiantes universitarios de Ecuador de áreas relacionadas con el bienestar y la salud. Universitas Psychologica, 16(4), 1-12. https://doi .org/10.11144/Javeriana.upsy16-4.spdv

\section{RESUMEN}

Este estudio tiene como objetivo analizar las relaciones entre las actitudes sexistas, los pensamientos distorsionados sobre la mujer y el uso de la violencia y los comportamientos de violencia con la pareja, en universitarios ecuatorianos de ramas relacionas con el bienestar y la salud. La muestra está conformada por 646 alumnos de tres universidades (424 alumnas y 222 alumnos). Se aplicaron los siguientes instrumentos: Versión modificada de la Escala de Tácticas para los Conflictos, Inventario de Sexismo Ambivalente, Inventario de Pensamientos Distorsionados y Escala de Deseabilidad Social. Para la detección de diferencias estadísticamente significativas, se aplicó el test de Mann Whitney y el test de Kruskal Wallis. Para estimar el efecto de la deseabilidad social en las respuestas, se realizaron diferentes regresiones lineales. Los resultados identifican un alto nivel de sexismo y de violencia ejercida y sufrida en la muestra de universitarios, aunque estos parecen estar influidos por la deseabilidad social. Este hecho cobra especial relevancia debido al papel que juegan estos profesionales en la detección y tratamiento de violencia en la pareja. Los resultados obtenidos indican la oportunidad de trabajar en intervenciones educativas en población universitaria para minimizar los pensamientos sexistas que pueden ser fundamento de la violencia de género.

Palabras clave

sexismo; violencia de género; Ecuador; universidad; parejas jóvenes.

\section{ABSTRACT}

This study aims to analyze the relationships between sexist attitudes, distorted thoughts about women, the use of violence and violent behaviors in couples. The study was carried out in Ecuadorian universities 
with students related to Welfare and Health studies. There were 646 students from three universities (424 men and 222 women). The following tests were applied: Modified version of the Scale Conflict Tactics, Ambivalent Sexism Inventory, Inventory of Distorted Thoughts and Social Desirability Scale. The Mann-Whitney and Kruskal Wallis tests were applied to detect statistically significant differences. In order to estimate the effect of social desirability on responses it was performed different linear regressions. The results identify high levels of sexism and perpetrated and suffered violence, although these results seemed to be influenced by social desirability. This becomes especially important due to the role played by these professionals in the detection and treatment of intimate partner violence. The results show the importance to work on educational interventions in universities to minimize sexist thoughts that cause violence against women.

Keywords

sexism; gender violence; Ecuador; university; young couples.

La violencia de género es un problema de salud pública a nivel global con importantes consecuencias para la salud de las mujeres (Bonavitta \& de Garay, 2011; Campbell, 2002; Ellsberg, Jansen, Heise, Watts, \& Garcia-Moreno, 2008). Profundizar sobre sus causas requiere una mirada interdisciplinar que incorpore diferentes aspectos del problema y que permita establecer estrategias eficaces para su erradicación (Laurent, Platzer, \& Idomir, 2013; World Health Organization, 2013a).

Diferentes posicionamientos teóricos han abordado la violencia de género (p. ej., Cunningham et al., 1998; Foa, Cascardi, Zoellner, \& Feeny, 2000; Redondo \& Andrés, 2004). Dentro de ellos, se diferencian aquellas teorías que subrayan la importancia de las variables intrapersonales, interpersonales y las que sitúan la causa de la violencia en el ámbito sociocultural (Boira, 2010). Estas últimas destacan la importancia de los comportamientos y las actitudes sexistas para explicar el comportamiento violento hacia la mujer. Desde las primeras aportaciones realizadas por autores como Allport (1954), quien se refirió al sexismo como una actitud antipática hacia la mujer que la sitúa en una posición inferior al hombre (Rodríguez \& Magalhães, 2013), el sexismo se reconoce a nivel mundial como una de las causas de desigualdades sociales.

Glick y Fiske $(1996,2001)$ propusieron el que denominaron modelo de sexismo ambivalente donde diferencian dos expresiones del fenómeno: el sexismo hostil y el sexismo benevolente. El primero caracteriza a la mujer como frágil y sumisa a partir de una supuesta inferioridad de las mujeres como grupo, justificando el control social que ejercen los hombres. El benevolente presupone también la inferioridad de las mujeres, pero expresando el deseo de los hombres de proteger a las mujeres. Se trataría de un tipo de prejuicio hacia ellas, basado en una visión estereotipada y limitada de la mujer, pero visto con un enfoque afectivo positivo (Berrocal, Cuadrado, Navas, Quiles del Castillo, \& Morera, 2011). Como señalan Chen, Fiske y Lee (2009), el sexismo es una construcción multidimensional y su presencia se ha vinculado con diferentes constructos y con diversos contextos. Se ha relacionado con el autoconcepto, el racismo y la sensibilidad intercultural (Garaigordobil \& Aliri, 2011a), con la posible influencia de variables familiares (Garaigordobil \& Aliri, 2011b), con valores religiosos (Glick, Lameiras, $\&$ Castro, 2002), como apoyo a comportamientos de violencia sexual (Sierra, Santos-Iglesias, Gutiérrez-Quintanilla, Bermúdez, \& BuelaCasal, 2010) o con la forma como influyen en los ideales románticos de pareja (Lee, Fiske, Glick, \& Chen, 2010).

Por otra parte, el sexismo se ha relacionado con diferentes variables como el sexo, la edad y la ira. En cuanto al sexo, algunos trabajos destacan las mayores puntuaciones obtenidas por los hombres (Lameiras \& Rodríguez, 2003; Lee, Pratto, \& Li, 2007; Travaglia, Overall, \& Sibley, 2009). En relación con la asociación de sexismo y edad, parece que las actitudes sexistas tienden a disminuir con la edad (Garaigordobil \& Aliri, 2012; Lameiras \& Rodríguez, 2003; Zakrisson, Aderzén, Lenell, \& Sandelin; 2012). Respecto a la ira, Garaigordobil (2015) indica relaciones positivas entre los dos.

La aparición de las actitudes sexistas cada vez a más temprana edad configura un marco de relaciones idóneo para el desarrollo de 
comportamientos de abuso y violencia en las relaciones de pareja que establecen adolescentes y jóvenes (de Lemus \& Ryan, 2010; DíazAguado, 2006; Rodríguez et al., 2008). De hecho, Rodríguez, Lameiras, Cabrera y Faílde (2010) señalan que las actitudes sexistas son más frecuentes en jóvenes que cursan educación secundaria frente a estudiantes universitarios de mayor edad. En el marco de la población universitaria resulta también importante el análisis de sus posibles actitudes sexistas, especialmente en áreas relacionadas con el bienestar y la salud, puesto que como futuros profesionales pueden tener una actuación directa en el ámbito de la violencia de género (p. ej., Macías et al., 2012).

En relación con la violencia en parejas jóvenes, González-Ortega, Echeburúa y Corral (2008) analizaron las variables que pueden estar más implicadas en el desarrollo de este comportamiento. Para estos autores, en parejas jóvenes es más frecuente la violencia psicológica que la física. En el caso de los hombres, la violencia es más habitual cuando se revelan actitudes positivas hacia la violencia o hubo experiencias violentas con parejas anteriores o se presentan algunas características de la personalidad como la impulsividad, la irascibilidad, la ausencia de empatía o la baja autoestima. También parece haber una mayor predisposición al comportamiento agresivo cuando el varón presenta alteraciones psicopatológicas como el abuso de alcohol y drogas o celos patológicos. En el caso de la víctima, la probabilidad de sufrir violencia es mayor cuando el emparejamiento es temprano, si aparecen déficits psicológicos como una baja autoestima, carencias afectivas o problemas de asertividad, si la red familiar y social de apoyo está ausente o si adoptan conductas de riesgo. Algunas de estas conductas son inicio temprano de relaciones sexuales, promiscuidad sexual, prácticas sexuales de riesgo (sin preservativos $\mathrm{y}$ sin control anticonceptivo), pertenencia a pandillas violentas o exposición reiterada a la violencia en la comunidad.

No obstante, distintos trabajos abordan la violencia sexual y de pareja entre adolescentes y parejas jóvenes en diferentes contextos y países (p. ej., Blázquez, Moreno, \& GarcíaBaamonde, 2009; Corral, 2009; Doroszewicz \& Forbes, 2008; Fernández-Fuertes \& Fuertes, 2010; González \& Santana, 2001; Mohand, Herrera, \& Carracedo, 2014; Muñoz-Rivas, Graña, O’Leary, \& González, 2007; Ortega, Ortega, \& Sánchez, 2008; Rey-Anacona, 2013; Vázquez \& Castro, 2008; Vizcarra \& Póo, 2011), y también los que relacionan la prevalencia esta violencia con el sexismo (Ferrer, Bosch, Ramis, Torres, \& Navarro, 2006; Rojas-Solís \& Carpintero, 2011).

Por otra parte, en el estudio del sexismo y de las relaciones de violencia en las relaciones interpersonales, es importante considerar la deseabilidad social. En la aplicación de pruebas de autoinforme en contextos de violencia, es difícil que los sujetos admitan este tipo de comportamientos y se reconozcan bien como víctimas o como agresores (Boira, del Castillo, Carbajosa, \& Marcuello, 2013; Boira, Carbajosa, \& Lila, 2014; Helfritz, Stanford, \& Conklin, 2006; van de Mortel, 2008). En la revisión bibliográfica realizada para este trabajo, son pocos los trabajos que hayan analizado la deseabilidad social en los estudios sobre sexismo y violencia en población joven (p. ej., Ferrer et al., 2006).

En Ecuador, son también escasos los estudios que han profundizado tanto en las características específicas de la violencia de pareja como en la influencia de las actitudes de sexismo (Boira, Carbajosa, \& Méndez, 2015; Goicolea, Öhman, Torres, Morrás, \& Edin, 2012). Sin embargo, la prevalencia del problema es grave. Según la Encuesta Nacional de Relaciones Familiares y Violencia de Género contra las Mujeres de 2011 (Instituto Nacional de Estadística y Censos de Ecuador, 2012), seis de cada 10 mujeres han vivido algún tipo de violencia de género en Ecuador. Y, de ellas, el 87.3 \% sufrió violencia dentro de la pareja.

Como consecuencia de lo anterior, el objetivo de este artículo es analizar las relaciones entre las actitudes sexistas, los pensamientos distorsionados sobre la mujer y el uso de la violencia y los comportamientos de violencia con 
la pareja, en los universitarios ecuatorianos de ramas relacionadas con el bienestar y la salud.

\section{Método}

\section{Participantes}

Para la realización del estudio, se obtuvo una muestra por conveniencia de 646 alumnos y alumnas de tres universidades de Ecuador. La población del estudio fueron estudiantes de nivelación, primer, segundo y tercer curso de la carrera de Medicina de la Universidad de Cuenca $(n=331)$, de Psicología de la Universidad Técnica del Norte $(n=129)$ y de Psicología de la Universidad Central de Ecuador $(n=186)$.

El estudio se llevó a cabo a lo largo del mes de octubre de 2014. La recogida de los datos se realizó en las aulas habituales de los estudiantes durante el tiempo lectivo. La participación fue voluntaria y antes de la aplicación de los cuestionarios se informó sobre los objetivos de la investigación y se solicitó el consentimiento de los estudiantes. Junto con la información recogida en los cuestionarios, se pidieron también datos de carácter sociodemográfico.

\section{Materiales y métodos}

Los instrumentos aplicados en este estudio fueron los siguientes:

Versión Modificada de la Escala de Tácticas para los Conflictos ([M-CTS]; Cascardi, Avery-Leaf, O'Leary, \& Slep, 1999)

La M-CTS contiene 18 ítems sobre una escala de tipo Likert con 5 opciones de respuesta desde 1 (Nunca) hasta 5 (Muy a menudo). Esta escala evalúa la presencia de negociación, agresión física grave, agresión física media y agresión psicológica en la relación tanto para la víctima como para el perpetrador (Muñoz-Rivas, Andreu, Graña, O'Leary, \& González, 2007). Para este estudio, se utilizan las puntuaciones de las tres subescalas que valoran la presencia de agresión. Los $\alpha$ de Cronbach varían entre 0.64 y 0.81 para las subescalas referidas a la víctima y entre 0.62 y 0,81 para las referidas al perpetrador.

Inventario de Sexismo Ambivalente ([ASI]; Glick \& Fiske, 1996; adaptada al español por Expósito, Moya, \& Glick, 1998)

Escala de 22 ítems que evalúa el sexismo ambivalente. La escala valora el Sexismo Hostil $(\alpha$ de Cronbach $=0.91)$ y el Sexismo Benevolente $(\alpha$ de Cronbach $=0.85)$, a partir de una escala tipo Likert, con seis puntos de respuesta $(0=$ Totalmente en desacuerdo; $5=$ Totalmente de acuerdo). En ambos factores, a mayor puntuación mayores niveles de sexismo (el rango de puntuación es de 0 a 55 para cada factor). El $\alpha$ de Cronbach para la escala total es 0.91 .

Inventario de Pensamientos Distorsionados sobre la Mujer y el Uso de la Violencia ([IPDMV]; Echeburúa \& Fernández-Montalvo, 1998)

Se trata de un listado de 29 ítems de respuesta dicotómica que identifica los pensamientos irracionales del maltratador. De estos ítems, 13 se refieren a pensamientos distorsionados sobre la mujer y 16 al uso de la violencia como forma aceptable de resolver conflictos. Para este trabajo se ha considerado el número de pensamientos distorsionados presentes en cada individuo.

Escala de Deseabilidad Social (Crowne \& Marlowe, 1960, adaptación española de Ferrando \& Chico, 2000)

Esta escala evalúa la tendencia a presentarse a sí mismo de una forma socialmente deseable. El formato de respuesta es de verdadero $(\mathrm{V}=1) \mathrm{o}$ falso $(F=0)$. Para obtener la puntuación de cada sujeto, se suman las respuestas verdaderas siendo necesario invertir los ítems que se presentan en sentido negativo. A mayor puntuación, mayor presencia de deseabilidad social en la forma de presentarse el sujeto. El coeficiente de fiabilidad $\alpha$ de Cronbach es para esta escala de 0.78.

Previamente a la realización de los análisis estadísticos, se utilizó el M-CTS para clasificar a los participantes en función de si han ejercido/ sufrido violencia hacia/por parte de su pareja y según tipo de violencia. En primer lugar, se valoró el nivel alcanzado por cada sujeto para cada uno de los tipos de violencia que mide el cuestionario (física grave, media y violencia 
psicológica). Se utilizó la mediana para valorar el nivel de violencia en cada una de las variables, considerando que el participante sufre o ejerce violencia significativa si su puntuación queda por encima de la misma. En el caso del grupo de agresión física grave y de agresión física media, se asignó a los sujetos en función de la puntuación más alta obtenida en cada una de las dos subescalas. En el caso del grupo de violencia psicológica, estuvo formado únicamente por aquellos sujetos que puntuaron en la subescala de agresión psicológica y no en las subescalas de agresión física. Del mismo modo, se clasificó a los sujetos según su nivel de deseabilidad social, alta o baja según quedasen por encima o por debajo de la mediana. Se crearon lo siguientes grupos: respecto a la violencia ejercida se clasificó a los estudiantes en los siguientes grupos: a) ejercieron violencia física grave, b) ejercieron violencia física media, c) solo ejercieron violencia psicológica y d) no han tenido comportamientos violencia. En el mismo sentido, en cuanto a la violencia sufrida, los grupos fueron: a) sufrieron violencia física grave, b) sufrieron violencia física media, c) solo sufrieron violencia psicológica y d) no han sufrido comportamientos violencia. Finalmente, en relación con la deseabilidad: a) puntuación baja y b) puntuación alta.

Para el análisis de los datos, se realizó un estudio descriptivo de las variables sociodemográficas desagregado por sexo y de las puntuaciones obtenidas en las pruebas aplicadas. Asimismo, se analizaron las actitudes sexistas y los pensamientos distorsionados sobre la mujer y el uso de la violencia según el tipo de violencia ejercida o sufrida. Para la detección de diferencias estadísticamente significativas, se aplicaron pruebas no paramétricas al no haber un ajuste a la normalidad. En función del número de grupos se utilizó el test de Mann-Whitney o el test de Kruskal Wallis. Para estimar el efecto de la deseabilidad social en las respuestas sobre violencia en la pareja, se realizaron diferentes regresiones lineales, siendo la variable dependiente violencia sufrida, violencia ejercida contra la pareja, sexismo benévolo y sexismo hostil y la variable independiente la deseabilidad social. Este análisis se realizó desagregado por sexo.

\section{Resultados}

Respecto a las características sociodemográficas de los estudiantes, la edad media es de 19.82 años $(D E=2.4)$, con un rango que oscila entre los 17 y los 37 años. El $34.4 \%$ eran hombres $(n=222)$ y el $65.6 \%$ mujeres $(n=424)$. En cuanto al estado civil de los participantes, la mayoría de ellos, el $92.4 \%(n=597)$ están solteros. Asimismo, un porcentaje muy elevado se consideran mestizos $(94.4 \% ; n=610)$, frente a afroecuatorianos $(2.2$ $\% ; n=10)$ o indígenas $(1.5 \% ; n=10)$. Por último, el $46.7(n=302)$ reconoce tener pareja en el momento de la realización del estudio.

En la Tabla 1, se presentan las puntuaciones obtenidas por hombres y mujeres tras la aplicación de las pruebas. Los resultados muestran que hay diferencias significativas respecto a las puntuaciones en sexismo hostil (que es mayor en los hombres), pero no en sexismo benévolo. En cuanto a los pensamientos sobre el uso de la violencia, se observa que los hombres presentan mayores puntuaciones que las mujeres.

En relación a la violencia ejercida, son significativas las diferencias en las variables argumentación hacia la pareja y violencia física grave, siendo los hombres los que presentan puntuaciones más altas. En violencia sufrida, se producen diferencias significativas en argumentación desde la pareja, violencia psicológica y violencia física grave, siendo también los hombres los que identifican mayores niveles de violencia de ellas hacia ellos. 


\section{TABLA 1}

Comparación por sexo de las puntuaciones en el Inventario de Sexismo Ambivalente, Cuestionario de pensamientos distorsionados y CTS-M

\begin{tabular}{lllll} 
Item & $\begin{array}{c}\text { Hombres } \\
\text { (mean } \\
\text { Rank) }\end{array}$ & $\begin{array}{c}\text { Mujeres } \\
\text { (mean } \\
\text { Rank) }\end{array}$ & Media & $p$ valor \\
\hline Puntuación Sexismo hostil & 408.1 & 279.2 & 29.1 & $<0.005$ \\
Puntuación Sexismo benévolo & 325.7 & 322.3 & 31.4 & 0.825 \\
Deseabilidad social & 323.3 & 323.6 & 17.6 & 0.984 \\
Pensamientos distorsionados sobre la mujer & 328.9 & 320.7 & 4.2 & 0.586 \\
Pensamientos distorsionados sobre el uso de la violencia & 360.8 & 303.9 & 7.1 & \\
CTS-M Argumentación hacia la pareja & 365.6 & 301.5 & 9.77 & $<0.005$ \\
CTS-M Violencia Psicológica ejercida hacia la pareja & 308.1 & 331.6 & 14.6 & 0.129 \\
CTS-M Violencia Fisica Media ejercida hacia la pareja & 305.6 & 332.9 & 10.7 & 0.069 \\
CTS-M Violencia Fisica Grave ejercida hacia la pareja & 338.2 & 315.8 & 23 & 0.023 \\
CTS-M Argumentación sufrida por la pareja & 359.6 & 304.6 & 9.3 & $<0.005$ \\
CTS-M Violencia Psicológica suffida por la pareja & 360.6 & 304.1 & 13.9 & $<0.005$ \\
CTS-M Violencia Fisica Media sufrida por la pareja & 341.25 & 314.2 & 10.4 & 0.071 \\
CTS-M Violencia Fisica Grave sufrida por la pareja & 337.4 & 316.3 & 3.2 & $<0.005$
\end{tabular}

Nota:La prueba de contraste utilizada es el Test de Mann-Whitney U.

Fuente: elaboración propia.

En la Tabla 2, se muestra la mediana, que ha sido utilizada para la conformación de los grupos de estudiantes según el tipo de violencia ejercida o sufrida. Un $41.3 \%$ de los participantes ha ejercido violencia física media, mientras que un $36.4 \%$ la ha sufrido. En cuanto a la violencia física grave, fue ejercida por un $7.4 \%$ y sufrida por un $10.5 \%$ de los encuestados. Respecto a la violencia psicológica fue ejercida por un $15.2 \%$ y sufrida por un $13.8 \%$.

\section{TABLA 2}

Conformación de los grupos según el tipo de violencia ejercida o sufrida utilizando la mediana de las puntuaciones en el test CTS-M como punto de corte

\begin{tabular}{lcccccccc}
\hline & Tipo de violencia ejercida & \multicolumn{5}{c}{ Tipo de violencia suffida } \\
\cline { 2 - 9 } & Psicógica & Fisica nedia & Fisica grave & No violencia & Psicologica & Fisia Media & Fisica grave & No Violencia \\
\hline Mediana & 15 & 8 & 3 & - & 14 & 8 & 3 & - \\
$n(\%)$ & $98(15.2)$ & $267(41.3)$ & $48(7.4)$ & $233(36.1)$ & $89(13.8)$ & $235(36.4)$ & $68(10.5)$ & $254(39.3)$ \\
\hline
\end{tabular}
Fuente: elaboración propia.

Respecto a los cruces según el tipo de violencia ejercida y sufrida por los estudiantes, en la Tabla 3 se muestran los resultados que indican la existencia de relaciones estadísticamente significativas tanto con las puntuaciones obtenidas en la escala de sexismo (hostil y benévolo) como en relación a los pensamientos distorsionados sobre la mujer y el uso de la violencia. A medida que la intensidad de la violencia es mayor las puntuaciones de las variables analizadas son más altas.

TABLA 3

Puntuaciones en sexismo y pensamientos distorsionados según la violencia ejercida y sufrida

\begin{tabular}{|c|c|c|c|c|c|c|c|c|c|c|}
\hline & & & & & & 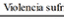 & & & & \\
\hline & No vorenent & Psso. & Fiska nocda & Fiska gave & $p$-valor & $\begin{array}{l}\text { Nov vilenciu } \\
\end{array}$ & Psisco. & Fisica nedia & Fisia grave & $p$-valor \\
\hline 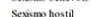 & 20662 & 280.5 & 357.6 & 344.5 & o & 295.7 & 295.8 & 355.2 & 344.11 & \\
\hline & 280.1 & 31999 & 355. & 344.7 & & 271.2 & 331.5 & 3625 & 373.8 & 0 \\
\hline $\begin{array}{l}\text { Dstorsonese } \\
\text { sobre hamer }\end{array}$ & 292.6 & 312.5 & 345 & 376.2 & 0.002 & 290.1 & 298.8 & 354.4 & 373.7 & 0 \\
\hline $\begin{array}{l}\text { sobre uso de } \mathrm{b} \\
\text { vojkciu }\end{array}$ & 297.9 & 300.7 & 34.1 & 378.3 & 0.000 & 2833 & 342.9 & 34.2 & 376.8 & 0 \\
\hline
\end{tabular}

Nota:la prueba de contraste utilizada es el Test de Kruskal Wallis.

Fuente: elaboración propia.

En la Tabla 4, se muestran los resultados del análisis de regresión considerando la posible influencia de la deseabilidad social en las respuestas sobre los diferentes tipos de violencia, tanto ejercida como sufrida, en hombres y mujeres. Se observa que a excepción de la violencia física grave y el sexismo benévolo, a mayor puntuación de deseabilidad mayor puntuación en las subescalas de violencia y sexismo.

\section{TABLA 4}

Análisis de regresión lineal simple en hombres y mujeres para analizar la influencia de la deseabilidad social en las escalas de violencia y sexismo aplicadas

\begin{tabular}{llllc} 
& Hombres & \multicolumn{3}{c}{ Mujeres } \\
\cline { 2 - 5 } & Coef. & $p$.valor & Coef. & $p$.valor \\
\hline Violencia psicológica sufrida & -0.245 & $<0.001$ & -0.288 & $<0.001$ \\
Violencia fisica media sufrida & -0.243 & 0.001 & -0.329 & $<0.001$ \\
Violencia física grave sufrida & -0.069 & 0.019 & -0.059 & $<0.001$ \\
& & & & \\
Violencia psicológica contra la pareja & -0.243 & $<0.001$ & -0.216 & $<0.001$ \\
Violencia fisica media contra la pareja & -0.365 & $<0.001$ & -0.25 & $<0.001$ \\
Violencia fisica grave contra la pareja & -0.014 & 0.615 & -0.028 & 0.52 \\
Sexismo benévolo & 0.37 & 0.198 & -0.193 & 0.036 \\
Sexismo hostil & -0.531 & $<0.001$ & -0.482 & $<0.001$ \\
\hline
\end{tabular}

Fuente: elaboración propia.

\section{Discusión}

En el presente artículo, se han analizado las relaciones entre las actitudes sexistas, los pensamientos distorsionados y la violencia en las relaciones de pareja, en estudiantes universitarios en Ecuador. Los resultados muestran diferencias significativas entre hombres y mujeres en cuanto a sexismo hostil, siendo este mayor en hombres que en mujeres; sin embargo, el sexismo benevolente se presenta en igual 
proporción. En cuanto a la relación del tipo de violencia ejercida o sufrida se encuentran diferencias estadísticamente significativas en las puntuaciones de sexismo (hostil y benevolente) y los pensamientos distorsionados sobre la mujer y el uso de la violencia.

Los resultados identifican un alto nivel de sexismo en la de universitarios ecuatorianos. Asimismo, confirman que son los hombres los que presentan un mayor nivel de sexismo hostil hacia las mujeres. Podría llamar la atención que no haya diferencias en sexismo benevolente entre hombres y mujeres, aunque este resultado ya se ha obtenido en anteriores estudios (Barreto \& Ellemers, 2005; Peña, Ramos, \& Luzón, 2011; Rodríguez \& Magalhães, 2013). Este sexismo disfrazado de falso reconocimiento y afecto, muy presente en las y los estudiantes, refuerza el rol tradicional de la mujer, justificando las actitudes que implican violencia (Rodriguez \& Lameiras, 2002).

Como puede observarse en los resultados, las altas puntuaciones en sexismo benévolo se relacionan con la presencia de violencia psicológica y de violencia física (moderada y grave), tanto ejercida como sufrida. En el caso de sexismo hostil, según la perspectiva feminista, la violencia en la pareja es la consecuencia extrema de las estructuras de poder, fuertemente asimétricas, donde los hombres ocupan las posiciones de poder y las mujeres adoptan una posición subordinada. Los resultados también muestran una relación altamente significativa entre el sexismo hostil y la gravedad de la violencia.

En el contexto latinoamericano, el marianismo, refuerza el constructo de las mujeres como sumisas, castas y sacrificadas, a manera de la imagen cristiana de María (Goicolea et al., 2012). Los resultados revelan que se necesita eliminar en los universitarios, incluso entre las mismas féminas, la imagen de una mujer estereotipada y limitada a roles tradicionales. El marianismo es una vertiente de pensamiento que genera sexismo ambivalente, dependiendo de su interacción con el contexto cultural y educativo.

Es importante destacar que los hombres son los que más habitualmente ejercen violencia, pero también son los que perciben violencia en mayor frecuencia. Esta situación ha sido explorada previamente en población universitaria (Vizcarra \& Póo, 2011). Allen, Swan y Raghavan (2009) sugieren que aunque mujeres y hombres ejercen y sufren violencia en sus relaciones, el contexto es diferente, ya que la violencia ejercida por la mujer suele ser como respuesta a la violencia masculina ejercida previamente (Graves, Sechrist, White, $\&$ Paradise, 2005; Straus, 2010). Igualmente, la sufrida en el caso de las mujeres tiene una mayor probabilidad de producir mayor daño físico y psicológico (Allen et al., 2009).

En Ecuador, ha habido un trabajo intenso desde las instituciones y organizaciones sociales sobre la concienciación en temas de violencia de género y violencia en la pareja, especialmente en población joven (Congreso Nacional del Ecuador, 1995; Jubb et al., 2008). Sin embargo, queda todavía mucho por hacer para erradicar los estereotipos de género y disminuir la prevalencia de la violencia contra las mujeres. En este sentido, el hecho de que en población universitaria, y por tanto en futuros profesionales, se detecten actitudes relacionadas con el sexismo hostil es preocupante y cobra especial relevancia en estudiantes de áreas relacionadas con el bienestar y la salud. Estos profesionales juegan un papel importante en la detección y tratamiento de casos de violencia de género (World Health Organization, 2013b). Las mujeres que sufren violencia a menudo buscan atención médica, aunque no revelen que la consulta esté asociada al abuso o violencia. El médico u otros profesionales orientados al bienestar de las personas como psicólogos o trabajadores sociales pueden ser el primer contacto de aquellas mujeres que se encuentran viviendo una situación de violencia y quizás en quienes confíen para revelar su situación (WHO, 2013b). Es posible que los estereotipos sexistas que pueden presentarse en los y las profesionales lleven a justificar la actitud y los comportamientos de violencia del hombre.

Por otra parte, el estigma asociado a reconocerse como víctima o a admitir comportamientos violentos puede interferir en las respuestas a los cuestionarios que se han planteado en este estudio. Por ello, se 
incluyó una medida de deseabilidad social para tratar de valorar dicha influencia. El análisis de regresión realizado muestra la influencia de la deseabilidad social en los cuestionarios de autoinforme aplicados. Tanto hombres como mujeres con deseabilidad baja mostraron mayor probabilidad de haber respondido afirmativamente a preguntas sobre violencia ejercida y sufrida. Una posible explicación a este hecho correspondería a una mayor sinceridad en las respuestas de los sujetos que obtuvieron las puntuaciones más altas en sexismo y violencia y, por el contrario, un mayor ocultamiento de este tipo de actitudes y comportamientos en estudiantes que contestaron de una forma más socialmente deseable. En cualquier caso, la deseabilidad social introduce limitaciones en las respuestas e interpretación de los resultados y, en términos de investigación, hace necesario implementar diseños complementarios de valoración de estos comportamientos que incorporen estrategias cualitativas para contrarrestar el efecto de una respuesta socialmente deseable. Además, los cambios sociales de los últimos años han hecho más difusas las fronteras del sexismo, por ello, se debe realizar una evaluación sutil de lo que se consideran actitudes sexistas. Estudios previos indican que el alumnado sin formación específica sobre el tema muestra creencias y actitudes menos críticas sobre la violencia en la pareja.

Finalmente, el presente estudio cuenta con ciertas limitaciones. En primer lugar, se trata de una muestra seleccionada por conveniencia, no representativa, lo que limita la extrapolación de datos a la población universitaria general. En segundo lugar, consiste en un estudio transversal que, aunque establece relaciones claras entre sexismo, pensamientos distorsionados y comportamientos de violencia, no permite establecer relaciones causa-efecto. Sin embargo, los resultados muestran interés al hacer referencia a estudiantes de primeros años relacionados con el área de bienestar (psicología y medicina), futuros profesionales con responsabilidades ligadas a la detección y tratamiento de violencia en la pareja. La identificación de actitudes sexistas en este grupo, para justificar las agresiones, puede condicionar su práctica profesional. Por otro lado, la muestra se encuentra mayoritariamente formada por mujeres, evidenciando que es necesario trabajar también con ellas para eliminar estereotipos de género.

Para seguir adelante, es necesario destacar que los resultados obtenidos indican la oportunidad de trabajar en intervenciones educativas en población universitaria, para minimizar los pensamientos sexistas y de distorsión de la figura de la mujer, como forma de prevenir la violencia en las relaciones de pareja. Deben incorporarse al currículo universitario en general, y especialmente en el colectivo del área de salud, materias y contenidos transversales sobre esta temática, para evitar futuras agresiones y para mejorar la detección y tratamiento de este tipo de violencia.

\section{Agradecimientos}

Agradecemos de manera especial al Proyecto Prometeo de la Secretaría de Educación Superior Ciencia, Tecnología e Innovación de la República de Ecuador.

\section{Referencias}

Allen, C. T., Swan, S. C., \& Raghavan, C. (2009). Gender symmetry, sexism, and intimate partner violence. Journal of Interpersonal Violence, 24, 1816-1834. https:// doi.org/10.1177/0886260508325496

Allport, G. (1954). The nature of prejudice. Reading, MA: Addison-Wesley.

Barreto, M., \& Ellemers, N. (2005). The burden of benevolent sexism: How it contributes to the maintenance of gender inequalities. European Journal of Social Psychology, 35, 633-642. https://doi.org/10.1002/ejsp.270

Berrocal, C., Cuadrado, M. I., Navas, M. S., Quiles del Castillo, M. N., \& Morera, M. D. (2011). Sexismo hostil y benevolente: dimensiones de comparación intergrupal, imagen de los subtipos de mujer y 
autoimagen del endogrupo. Revista de Psicología Social, 26(1), 45-62.

Blázquez, M., Moreno, J. M., \& GarcíaBaamonde, M. E. (2009). Estudio del maltrato psicológico en las relaciones de pareja en jóvenes universitarios. Electronic Journal of Research in Educational Psychology, 7, 691-714.

Boira, S. (2010). Hombres maltratadores. Historias de violencia masculina. Zaragoza: Prensas Universitarias de Zaragoza.

Boira, S., Carbajosa, P., \& Lila, M. (2014). Principales retos en el tratamiento grupal de los hombres condenados por un delito de violencia de género. Clínica Contemporánea, 5, 1-13. https://doi.org/10.5093/cc2014a1

Boira, S., Carbajosa, P., \& Méndez, R. (2015). Miedo, conformidad y silencio. La violencia en las relaciones de pareja en áreas rurales de Ecuador. Psychosocial Intervention, 25(1), 9-17. https://doi.org/10.1016/j.psi.2015.07.008

Boira, S., del Castillo, M., Carbajosa, P., \& Marcuello, C. (2013). Context of treatment and therapeutic alliance: Critical factors in court-ordered batterer intervention programs. The Spanish Journal of Psychology, 16, 1-13. https:// doi.org/10.1017/sjp.2013.43

Bonavitta, P., \& de Garay, J. (2011). De estereotipos, violencia y sexismo: la construcción de las mujeres en los medios mexicanos y argentinos. Anagramas, 9(18), 15-30.

Campbell, J. C. (2002). Health consequences of intimate partner violence. The Lancet, 359(9314), 1331-1336. https:// doi.org/10.1016/S0140-6736(02)08336-8

Cascardi, M., Avery-Leaf, S., O'Leary, K. D., \& Slep, A. M. S. (1999). Factor structure and convergent validity of the conflict tactics scale in high school students. Psychological Assessment, 11, 546-555. https:// doi.org/10.1037//1040-3590.11.4.546

Chen, Z., Fiske, S. T., \& Lee, T. L. (2009). Ambivalent sexism and power-related gender-role ideology in marriage. Sex
Roles, 60, 765-78. https://doi.org/10.1007/ s11199-009-9585-9

Corral, S. (2009). Estudio de la violencia en el noviazgo en jóvenes universitarios/as: cronicidad, severidad y mutualidad de las conductas violentas. Psicopatología Clínica Legal y Forense, 9, 29-48.

Crowne, D. P., \& Marlowe, D. (1960). A new scale of social desirability independent of psychopathology. Journal of Consulting Psychology, 24, 349-354. https:// doi.org/10.1037/h00447358

Cunningham, A., Jaffe, P. G., Baker, L., Dick, T., Malla, S., Mazaheri, N., \& Poisson, S. (1998). Theory-derived explanations of male violence against female partners: Literature update and related implications for treatment and evaluation. Londres: Family Court Clinic.

Díaz-Aguado, M. J. (2006). Sexismo, violencia de género y acoso escolar. Propuestas para una prevención integral de la violencia. Revista de Estudios de Juventud, 73, 38-57.

Doroszewicz, K., \& Forbes, G. (2008). Experiences with dating aggression and sexual coercion among Polish college students. Journal of Interpersonal Violence, 23, 58-73. https:// doi.org/10.1177/0886260507307651

Echeburúa, E., \& Fernández-Montalvo, J. (1998). Hombres maltratadores. En E. Echeburúa \& P. Corral (Eds.), Manual de violencia familiar (pp. 112-113). Madrid: Siglo XXI.

Ecuador, Instituto Nacional de Estadística y Censos. (2012). Encuesta Nacional de Relaciones Familiares y Violencia de Género contra las Mujeres Noviembre 2011 (Documento DDI 2012-v1.3). Quito: Ministerio del Interior. Recuperado de http://anda.inec.gob.ec/anda/index.php/ catalog/94

Ecuador, Ley 103 de 1995. Ley Contra la Violencia a la Mujer y la Familia. Noviembre 14 de 1995. Registro oficial N. $\circ 839$.

Ellsberg, M., Jansen, H. A., Heise, L., Watts, C. H., \& Garcia-Moreno, C. (2008). Intimate 
partner violence and women's physical and mental health in the WHO multicountry study on women's health and domestic violence: An observational study. The Lancet, 371 (9619), 1165-1172. https:// doi.org/10.1016/S0140-6736(08)60522-X

Expósito, F., Moya, M., \& Glick, P. (1998). Sexismo ambivalente: medición y correlatos. Revista de Psicología Social, 13(2), 159-169.

Fernández-Fuertes, A., \& Fuertes, A. (2010). Physical and psychological aggression in dating relationships of Spanish adolescents: Motives and consequences. Child Abuse $\mathbb{E}$ Neglect, 34, 183-191. https:// doi.org/10.1016/j.chiabu.2010.01.002

Ferrando, P. J., \& Chico, E. (2000). Adaptación y análisis psicométrico de la Escala de Deseabilidad Social de Marlowe y Crowne. Psicothema, 12(3), 383-389.

Ferrer, V. A., Bosch, E., Ramis, C., Torres E. G., \& Navarro, C. (2006). La violencia contra las mujeres en la pareja: creencias y actitudes en estudiantes universitarios. Psicothema, 18(3), 359-366.

Foa, E., Cascardi, M., Zoellner, L., \& Feeny, N. (2000). Psychological and environmental factors associated with partner violence. Trauma, Violence $\mathcal{E}$ Abuse, 1, 67-91. https:// doi.org/10.1177/1524838000001001005

Garaigordobil, M. (2015). Sexismo y expresión de la ira: diferencias de género, cambios con la edad y correlaciones entre ambos constructos. Revista Argentina de Clínica Psicológica, 24(1), 35-42.

Garaigordobil, M., \& Aliri, J. (2011a). Sexismo hostil y benevolente: relaciones con autoconcepto, racismo y sensibilidad intercultural. Revista de Psicodidáctica, 16(2), 331-350.

Garaigordobil, M., \& Aliri, J. (2011b). Conexión intergeneracional del sexismo: influencia de variables familiares. Psicothema, 23(3), 382-387.

Garaigordobil, M., \& Aliri, J. (2012). Parental socialization styles, parents' educational level, and sexist attitudes in adolescence. Spanish Journal of Psychology,
15, 592-603. https://doi.org/10.5209/ rev_SJOP.2012.v15.n2.38870

Glick, P., \& Fiske, S. (1996). The Ambivalent Sexism Inventory: Differentiating hostile and benevolent sexism. Journal of Personality and Social Psychology, 70(3), 491-512. https:// doi.org/10.1037/0022-3514.70.3.491

Glick, P., \& Fiske, S. (2001). An ambivalent alliance: Hostile and benevolent sexism as complementary justifications of gender inequality. American Psychologist, 56, 109-118. https:// doi.org/10.1037/0003-066X.56.2.109

Glick, P., Lameiras, M., \& Castro, Y. (2002). Education and Catholic religiosity as predictors of hostile and benevolent sexism toward women and men. Sex Roles, 47, 433-441. https://doi.org/10.1023/ A:1021696209949

Goicolea, I., Öhman, A., Salazar Torres, M., Morrás, I., \& Edin, K. (2012). Condemning violence without rejecting sexism? Exploring how young men understand intimate partner violence in Ecuador. Global Health Action, 5. https:// doi.org/10.3402/gha.v5i0.18049

González, R., \& Santana, J. D. (2001). La violencia en parejas jóvenes. Psicothema, 13(1), 127-131.

González-Ortega, I., Echeburúa, E., \& Corral, P. (2008). Variables significativas en las relaciones violentas en parejas jóvenes: una revisión. Psicología Conductual, 16(2), 207-225.

Graves, K., Sechrist, S., White, J. W., \& Paradise, M. J. (2005). Intimate partner violence perpetrated by College Women within the context of a history of victimization. Psychology of Women Quarterly, 29, 278-289. https:// doi.org/10.1111/j.1471-6402.2005.00222.x

Helfritz, L., Stanford, M. S., \& Conklin, S. M. (2006). Usefulness of self-report instruments assessing men accused of domestic violence. The Psychological Record, 56, 171-180. 
Jubb, N., Camacho, G., D'Angelo, A., Yáñez, G., Hernández, K., \& Macassi, I. (2008). Regional mapping of women's police stations in Latin America. Quito: CEPLAES.

Lameiras, M., \& Rodríguez, Y. (2003). Evaluación del sexismo ambivalente en estudiantes gallegos/as. Acción Psicológica, 2, 131-136. https://doi.org/10.5944/ ap.2.2.526

Laurent, C., Platzer, M., \& Idomir, M. (2013). Femicide. A Global issue that demand action (Vol. 1). Viena: Acuns.

Lee, I., Pratto, F., \& Li, M. (2007). Social relationships and sexism in the United States and Taiwan. Journal of CrossCultural Psychology, 38(5), 595-612. https:// doi.org/10.1177/0022022107305241

Lee, T. L., Fiske, S. T., Glick, P., \& Chen, Z. (2010). Ambivalent sexism in close relationships: (Hostile) power and (benevolent) romance shape relationship ideals. Sex Roles, 62 (7-8), 583-601. https:// doi.org/10.1007/s11199-010-9770-x

Lemus de, S., \& Ryan, E. (2010). Relaciones de género en la infancia y adolescencia: desarrollo de las actitudes sexistas. En S. de Lemus \& E. Ryan (Coords.), Coeducación: propuestas para alcanzar la igualdad de género desde las aulas (pp. 69-100). Granada: Editorial Universitaria.

Macías, J., Gil, E., Rodríguez, M. A., González, J. R., González, M. del M., \& Soler, A. M. (2012). Creencias y actitudes del alumnado de Enfermería sobre la violencia de género. Index de Enfermería, 21(1-2). https:// doi.org/10.4321/ S1132-12962012000100003

Mohand, L. M., Herrera, L., \& Carracedo, S. (2014). Violencia de pareja en jóvenes estudiantes universitarios de diferente origen cultural. Revista de Educação e Humanidades, 5, 223-236.

Muñoz-Rivas, M. J., Graña, J. L., O'Leary, K. D., \& González, P. (2007). Physical and psychological aggression in dating relationships in Spanish university students. Psicothema, 19(1), 102-107.
Muñoz-Rivas, M., Andreu, J., Graña, J., O'Leary, D., \& González, M. (2007). Validación de la versión modificada de la Conflicts Tactics Scale (M-CTS) en población juvenil española. Psichotema, 19(4), 693-698.

Ortega, R., Ortega, F. J., \& Sánchez, V. (2008). Violencia sexual entre compañeros y violencia en parejas adolescentes. International Journal of Psychology and Psychological Therapy, 8(1), 63-72.

Peña, E., Ramos, E., \& Luzón, J. (2011). Proyecto de investigación, sexismo y violencia de género en la juventud andaluza. Resultados y recomentaciones. 26 Instituto Andaluz de la Mujer (en línea). http://www.uca.es/recursos/doc/uni dad_igualdad/47737780_1122011112236. pdf

Redondo, S., \& Andrés, A. (2004). Perfil y tratamiento del maltratador familiar. Cuadernos de la Guardia Civil: Revista de Seguridad Pública, 30, 25-36.

Rey-Anacona, C. A. (2013). Prevalencia y tipos de maltrato en el noviazgo en adolescentes y adultos jóvenes. Terapia Psicológica, 31 (2), 143-154.

Rodríguez, L., Antuña, A., López-Cepero, J., Rodríguez-Díaz, F. J., Herrero, F. J., \& Bringas, C. (2008). Una aproximación al estudio de las actitudes sexistas en novios adolescentes. En F. J. Rodríguez, C. Bringas, F. Fariña, R. Arce \& A. Bernardo (Orgs.), Psicología jurídica. familia y victimología (pp. 219-227). Oviedo: Ediciones Universidad de Oviedo.

Rodríguez, R., Lameiras, M., Cabrera, M. V., \& Faílde, J. M. (2010). Evaluación de las actitudes sexistas en estudiantes españoles/ as de educación secundaria obligatoria. Psychologia: Avances de la Disciplina, 4(1), 11-24.

Rodríguez, Y., \& Lameiras, M. (2002). Evaluación del sexismo moderno en adolescentes. Revista de Psicología Social, $17(2), 119-128$.

Rodríguez, Y., \& Magalhães, M. J. (2013). El sexismo moderno en estudiantes universitarios/as. AGIR - 
Revista Interdisciplinar de Ciências Sociais e Humanas, 1(2), 113-121.

Rojas-Solís, J. L., \& Carpintero, E. (2011). Sexismo y agresiones físicas, sexuales y verbales-emocionales, en relaciones de noviazgo de estudiantes universitarios. Electronic Journal of Research in Educational Psychology, 9, 541-564.

Sierra, J. C., Santos-Iglesias, P., GutiérrezQuintanilla, R., Bermúdez, M. P., \& Buela-Casal, G. (2010). Factors associated with rape-supportive attitudes: Sociodemographic variables, aggressive personality, and sexist attitudes. Spanish Journal of Psychology, 13(1), 202-209.

Straus, M. A. (2010). Thirty years of denying the evidence on gender symmetry in partner violence: Implications for prevention and treatment. Partner Abuse, 1, 332-363. https://doi.org/10.1.1.372.5578

Travaglia, L. K., Overall, N. C., \& Sibley, C. G. (2009). Benevolent and hostile sexism and preferences for romantic partners. Personality and Individual Differences, 47, 599-604. https:// doi.org/10.1016/j.paid.2009.05.015

van de Mortel, T. F. (2008). Faking it: Social desirability response bias in self-report research. Australian Journal of Advanced Nursing, 25 (4), 40-48.

Vázquez, V., \& Castro, R. (2008). “iMi novio sería capaz de matarme?" Violencia en el noviazgo entre adolescentes de la Universidad Autónoma Chapingo, México. Revista Latinoamericana de Ciencias Sociales, Niñez y Juventud, 6, 709-738.

Vizcarra, M. B., \& Póo, A. M. (2011). Violencia de pareja en estudiantes universitarios del sur de Chile. Universitas Psychologica, 10 (1), 89-98.

World Health Organization. (2013a). Global and regional estimates of violence against women: Prevalence and health effects of intimate partner violence and non-partner sexual violence (Informe N. ${ }^{\circ} \mathrm{WHO}$ : $\left.\begin{array}{lllll}978 & 92 & 4 & 156462 & 5\end{array}\right)$. Recuperado de http://www.who.int/reproductivehealth/ publications/violence/9789241564625
World Health Organization. (2013b). Responding to intimate partner violence and sexual violence against women: WHO clinical and policy guidelines. Ginebra: Autor.

Zakrisson, I., Anderzén, M., Lenell, F., \& Sandelin, H. (2012). Ambivalent sexism: A tool for understanding and improving gender relations in organizations. Scandinavian Journal of Psychology, 53, 64-70. https:// doi.org/10.1111/j.1467-9450.2011.00900.x

\section{Notas}

* Artículo de investigación. 